Computing and Informatics, Vol. 40, 2021, 815-832, doi: 10.31577/cai_2021_4_815

\title{
NEW SIMULATION SOFTWARE TECHNOLOGIES AT THE LHCB EXPERIMENT AT CERN
}

\author{
Michał MAZUREK \\ CERN, European Organization for Nuclear Reasearch \\ Esplanade des Particules 1, 1211 Meyrin, Switzerland \\ E3 \\ NCBJ, National Centre for Nuclear Research \\ Andrzeja Soltana 7, 05-400 Otwock, Poland \\ e-mail: michal.mazurek@cern.ch
}

\author{
Gloria Corti, Dominik Müller \\ CERN, European Organization for Nuclear Reasearch \\ Esplanade des Particules 1, 1211 Meyrin, Switzerland \\ e-mail: gloria.corti@cern.ch
}

\begin{abstract}
The LHCb experiment at the Large Hadron Collider (LHC) at CERN has successfully performed a large number of physics measurements during Runs 1 and 2 of the LHC. Monte Carlo simulation is the key to the interpretation of these and future measurements. The LHCb experiment is currently undergoing a major detector upgrade for Run 3 of the LHC to process events with five times higher luminosity. New simulation software technologies have to be introduced to produce simulated data samples of sufficient size within the computing resources allocated for the next few years. Therefore, the LHCb collaboration is currently preparing an upgraded version of its GaUss simulation framework. The new version provides the $\mathrm{LHCb}$ specific functionality while its generic simulation infrastructure has been encapsulated in an experiment independent framework, GaUssino. The latter combines the GAUDI core software framework and the GEANT4 simulation toolkit and fully exploits their multi-threading capabilities. A prototype of a fast simulation interface to the simulation toolkit is being developed as the latest addition to GAUSsino to provide an extensive palette of fast simulation models, including new deep learning-based options.
\end{abstract}


Keywords: LHCb, CERN, Monte Carlo simulation, fast simulations, multi-threading

Mathematics Subject Classification 2010: 68-04

\section{INTRODUCTION}

The LHCb detector is a single-arm spectrometer with a forward angular coverage, designed for the study of particles containing $b$ or $c$ quarks. It is located at the Large Hadron Collider (LHC) at the European Organization for Nuclear Research (CERN). During the LHC Run 1 and Run 2 the LHCb experiment has successfully performed a large number of measurements in heavy flavour physics, however, the precision of its measurements is becoming more and more limited by the statistics of the simulated samples. In addition, the LHCb experiment is currently installing a major upgrade for the data taking of Run 3 in order to be able to process events with a 5 times higher luminosity. The $\mathrm{LHCb}$ experiment is also planning a further upgrade with another increase in luminosity by a factor of 5 to 10 . Therefore, higher capacity in data storage and computing power is needed in order to prepare the experiment for the changes in the future Runs. Moreover, the whole software is being adapted to work in a multi-threaded environment in order to efficiently use the available computing resources. As an example, the experiment will rely on a real-time reconstruction trigger implemented on GPUs. In the last years, the LHCb GAUss simulation framework has been evolving to provide a palette of fast simulations options and to use the new functional GAUDI framework and GEANT4 multi-threading technologies. In this paper, recent developments in the simulation software are discussed. A brief description of the LHCb detector and its upgrade are presented in Section 2. Details about the $\mathrm{LHCb}$ simulation software and the new multi-threading approach can be found in Section 3. Finally, a description of the fast simulation interface in GAUSS-ON-GAUssino with an example on how to use the libraries needed to prepare a training dataset is presented in Section 4

\section{LHCB DETECTOR}

The LHCb detector [11, 12] covers the pseudorapidity range $2<\eta<5$. It includes a high-precision tracking system consisting of a silicon-strip vertex detector (VErtex LOcator, VELO) surrounding the $p p$ interaction region, a large-area siliconstrip detector (TT) located upstream of a dipole magnet with a bending power of about $4 \mathrm{Tm}$, and three stations of silicon-strip detectors and straw drift tubes placed downstream of the magnet. The tracking system (T1-T3) provides the momentum measurement of charged particles. Different types of charged hadrons are distinguished using information from two ring-imaging Cherenkov detectors, located upstream and downstream of the magnet (RICH1 and RICH2). Photons, electrons 
and hadrons are identified by the LHCb calorimeter system [1. An Electromagnetic CALorimeter (ECAL) and a Hadronic CALorimeter (HCAL) determine the amount of energy deposited by the particles of electromagnetic or hadronic nature, respectively. Some minimum ionizing particles may also be registered by the calorimeters. Muons are identified by a system composed of alternating layers of iron and multiwire proportional chambers (M1-M5). In order to reduce the number of events collected and maximizing those of interest at the same time, an online event selection is performed via a combination of hardware and software triggers. The L0 trigger (hardware stage) operates on the information coming from the calorimeter and muon systems at a rate of $40 \mathrm{MHz}$, followed by a High Level Trigger (HLT, software stage), which applies a full event reconstruction.

The LHCb collaboration is currently installing a major upgrade that will allow the experiment to significantly increase the amount of data collected, by operating with a luminosity of up to $2 \times 10^{33} \mathrm{~cm}^{-2} \mathrm{~s}^{-1}, 5$ times higher than that of Run 1 and Run 2 and writing data at a $30 \mathrm{MHz}$ rate. In order to cope with the higher readout rates, the front-end electronics of all the sub-detectors will be replaced. Moreover, most of the LHCb sub-detectors will be replaced and only the heaviest ones, namely the calorimeters and muon stations, will remain mostly in place. In Run 1 and Run 2 the event output rate was limited to $1 \mathrm{MHz}$ and through a hardware-based L0 trigger. This has been removed for Run 3 and LHCb is introducing a software-only trigger working at the $30 \mathrm{MHz}$ bunch crossing rate. Processing and the selection of events at this very high rate have a major impact on software and computing systems.

\section{SIMULATION SOFTWARE AT LHCB}

In $\mathrm{LHCb}$, data collected by the detector is processed using a set of custom applications based on the GAUDi [4, 8] core software framework. A simplified view on the LHCb data processing applications, as used in Run 1 and Run 2, is shown in Figure 1. LHCb applications identically process events either collected by the detector itself or events produced by the simulation software. Simulated events are first handled by GAuss that performs the event generation and tracking of particles through the detector material. The Boole application mimics the specific subdetector technologies and electronics response, providing the same digital output of the data acquisition system. The MoORE application then emulates the L0 trigger response for the simulated data and provides the software-based HLT trigger. More complex objects are produced by the offline reconstruction in BRUNEL. Each application reads and writes processed data to storage.

\subsection{GaUss Simulation Software}

The LHCb simulation framework Gauss [7] is built, as all the other LHCb software applications, using the general GAUDI data-processing framework. GAUDI helps 


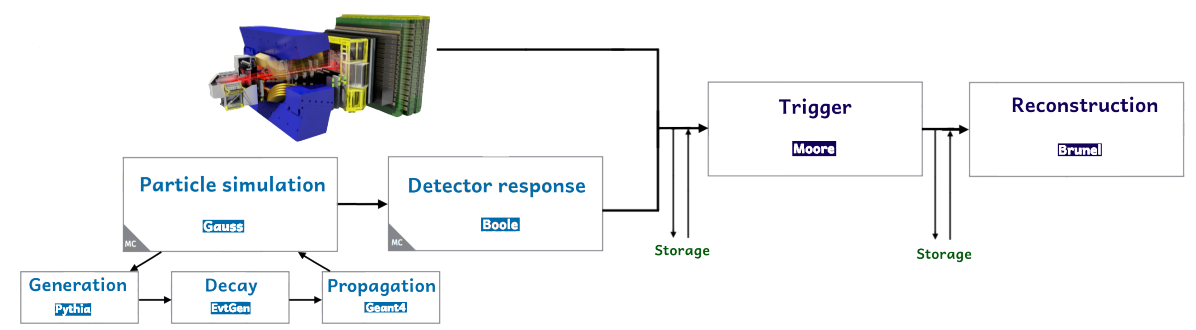

Figure 1. Simplified view on the $\mathrm{LHCb}$ data flow and data processing applications, as used in Run 1 and Run 2

in the configuration of algorithms and tools in the application. It also controls the data flow and the event loop. Dependencies in the simulation framework are illustrated in Figure 2 a), One of the two main tasks of the GAUss framework is to control the generation of collisions, in most cases $p p$ with PyтhiA [24, 25], where a specific LHCb configuration [5] is used. Gauss then makes use of EvTGEN [10] in order to model the decays of unstable particles. The other main task of GAUss is the propagation of the generated particles through the experimental apparatus and the simulation of physics processes occurring within the sub-detectors using the GEANT4 toolkit [3, 2, 7]. The information to be able to mimic the response of given sub-detectors when a particle intersects them (MC hits) and to understand trigger and reconstruction performance ( $\mathrm{MC}$ truth) are written to a file for further processing.

\subsection{LHCb Software Changes for Run 3}

As mentioned in the first section, providing a high-throughput software relying on a better utilization of modern computing architectures is crucial in order to cope with the higher event output rate. A typical $\mathrm{LHCb}$ application needs around 2 to $4 \mathrm{~GB}$ RAM on average [6]. Therefore, a multi-process approach, where each instance of the application is run on a separate core, is not optimal from the memory consumption point of view. As a result, the LHCb core software framework, GAUDI, has been reengineered to work in a multi-threading mode in order to benefit from the inter-event parallelism as much as possible. A lot of effort has been put in order to guarantee thread-safety, as many objects in the application are now shared between the events. This has been achieved mostly by introducing a functional paradigm, in which a set of algorithms operate on data by either producing, transforming or consuming it, and ensuring that the internal state is unchanged. The implementation of a new scheduler [18] and the optimization of the software by utilizing vector processing units, introducing new $\mathrm{C}++$ features and rethinking the data models, were the other key components in the new GAUDI framework.

Gauss, the LHCb simulation software, like all the other LHCb applications, depends on the changes introduced in GaUdi. Moreover, Gauss has to adapt to the 


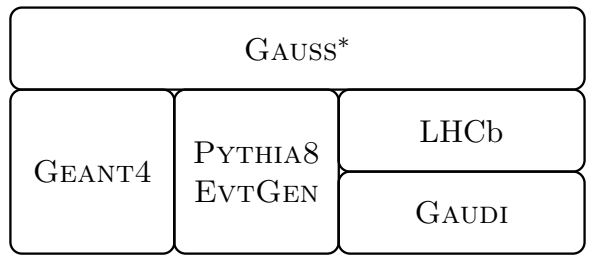

a) Gauss Run 1 and Run 2 framework

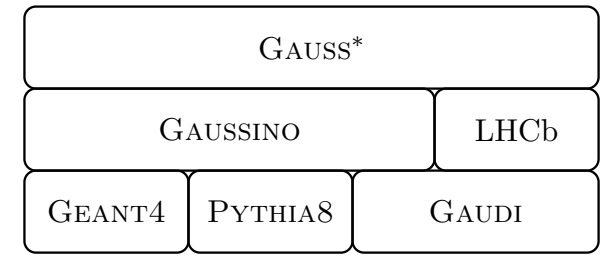

b) Gauss-on-Gaussino framework

* Additional LHCb-specific configuration for Geant4, Pythia and EvtGen is not shown in the dependency graph.

Figure 2. Dependencies [17] in the simulation software stack before and after upgrade

changes introduced by the new approach in the detector description. DD4HEP [9] is a detector description toolkit that provides experiment-independent libraries for conditions management and visualization of the geometry. On the other hand, Gauss still has to provide support for the older geometries when producing simulated samples for Run 1 and Run 2 physics analyses. Additional challenge arises from the fact that GAuss delegates tasks to external libraries used in the framework (e.g. GEANT4) that are already working in their own multi-threading realms.

\subsection{Gauss-on-Gaussino}

In order to address the challenges mentioned above, the LHCb simulation team decided to move all the LHCb-independent components from the simulation software and place it in a separate project, called Gaussino [23, 19, 15], as a core simulation framework, on which all the new versions of GAUss will be built. It is also possible to run Gaussino as a standalone application. Dependencies in the new LHCb version of the framework, called here GAUSS-ON-GAUssino for clarity, are illustrated in Figure 2b). GAUssino follows the GAUDI's inter-event-based parallelism of the event loop, in which algorithms are scheduled in a way that guarantees threadsafety. Gaussino communicates with Geant4 objects by creating corresponding factories that act as GAUDI tools. In the event loop, GAUDI places the generated event on the top of a FIFO queue. GEANT4 worker threads then take the event from the queue and perform the simulation following their own multi-threading scheme. The multi-threaded approach in GAUSS-ON-GAussino has already made it possible to simulate more events in time by limiting the memory consumption of each event as shown in an earlier paper [15]. Nevertheless, this will not be enough to meet the requirements imposed by the upgrade of the detector, which will be discussed in the following section. 


\section{FAST SIMULATION INTERFACE IN GAUSS-ON-GAUSSINO}

Producing necessary simulated samples for physics analyses with GAUss consumed around $80 \%$ [14, 26, 13] of all the distributed computing resources available to LHCb during Run 2. An additional few percent were used to process the simulated samples through the subsequent applications. The increase in the number of events, caused by the upcoming upgrade, will require to simulate even more events. If all samples would be produced with a detailed simulation, the foreseen computing resources allocated to $\mathrm{LHCb}$ will be quickly exceeded (Figure 3). Scenarios with different fractions of the samples produced with detailed simulation, fast, and ultrafast models would allow to fulfill the needs within the resource forecast.

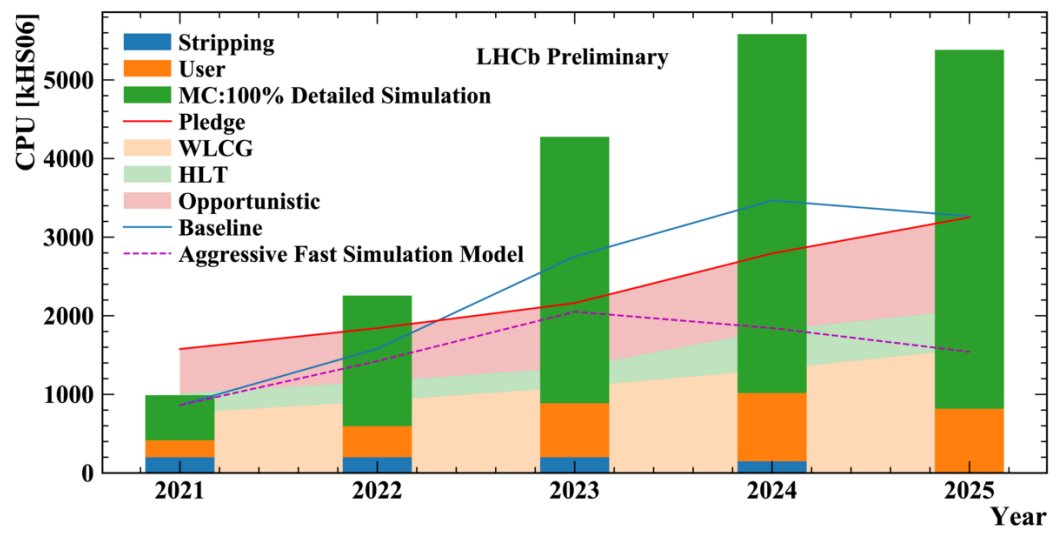

Figure 3. Projection of the computing resources available to LHCb [14]. Monte Carlo simulations will quickly exceed in the upcoming years the resources allocated to LHCb by the distributed computing system provided that no action is taken in order to fit in the requirements (e.g. fast simulations). The CPU needs for producing $100 \%$ of the required samples with the detailed simulation is shown by the green bars. The CPU needs in two scenarios with different fractions of events produced with detailed, fast and ultra-fast simulations are shown by the blue and dashed purple lines, respectively.

A lot of efforts have been made by the Gauss developers in order to reduce the CPU time spent in the simulation. In GAUss, propagating particles through matter dominates the time spent by the application. A finer analysis of the relative time spent in each sub-detector of the upgrade geometry is presented in Figure $4 \mathrm{a}$ ) for the current version of the framework. As seen in the results, simulation of the particle showers in the calorimeters is especially demanding. Consistent results are obtained for GAUSS-ON-GAUSSINO as expected and shown in Figure 4 b). Additional information about the time spent by each particle is given in Figure 10 and Figure 11 in the Appendix.

A campaign with the goal to introduce a palette of fast simulation models to complement the detailed GeAnt4-based simulation has been launched. ReDecay [20] 
is a technique, in which the underlying $p p$ interaction is reused in the simulation of the detector multiple times, with an independently generated signal decay for each event. Lamarr [16] is an in-house, ultra-fast parametrization framework that extends up to the reconstruction level and provides high-level reconstruction objects in the output. When it comes to the calorimeters, a fast simulation model of ECAL based on a point library [21] is currently being implemented. There are already a few proposals of fast simulation models based on generative adversarial networks 22] for the calorimeters and RICH detectors, however, a special interface is needed in GAUSSINO to exploit them via the fast simulation mechanisms available in GEANT4. Recent work to support the implementation of these last fast simulations in Gaussino is described in the rest of this section.

\subsection{Implementation of the Interface}

Gaussino provides a generic FastSimulation interface to Geant4 objects in order to minimize the work spent in the future on implementing fast simulation models, and also to guarantee the integrity of the simulated data. Following the convention already present in GAUSSINO, the FASTSImUlation interface consists of factories ensuring GEANT4 objects are configured properly and at the right moment when running the application. A set of the most important factories and their GEANT4 counterparts are presented in Figure 5. Most of the work necessary to implement a fast simulation model itself can be pushed to the configuration in python files. The most optimistic scenario is that the developer will only have to implement a G4VFastSimulationModel: :DoIt() callback method in $\mathrm{C}++$ that is the key component of the whole interface and actually describes the whole process of generating fast hits.

Two, purely abstract, fast simulation models are introduced in GAUssino in order to measure the performance of the interface, and to mark off a lower bound for all further fast simulation models. An ImmediateDeposit model generates one hit per particle that intercepts the region where the ImmediateDeposit model is active and deposits all of its energy in that hit. ImmediateDeposit gives useful information about the timing needed for the infrastructure itself to call the fast simulation methods. The ShowerDeposit model works in a similar manner, but it splits the energy of a particle into a selected number of hits, and generates them randomly around the position where the particle intercepted the region. ShowerDeposit provides the minimum amount of time needed to generate a specific number of hits with no additional calculations.

A comparison between different fast simulation models tested with a particle gun that creates a grid of 3328 evenly-spaced photons originating at the $\mathrm{LHCb}$ interaction point, is presented in Figure 6. Time spent in the ImmediateDeposit model is comparable across different photon energies and works as expected. On the other hand, the time spent in the ShowerDeposit model increases with the energy of photons. Naturally, this is caused by a larger number of hits generated in each shower. In the worst case, a $100 \mathrm{GeV}$ photon generates 21558 hits on aver- 


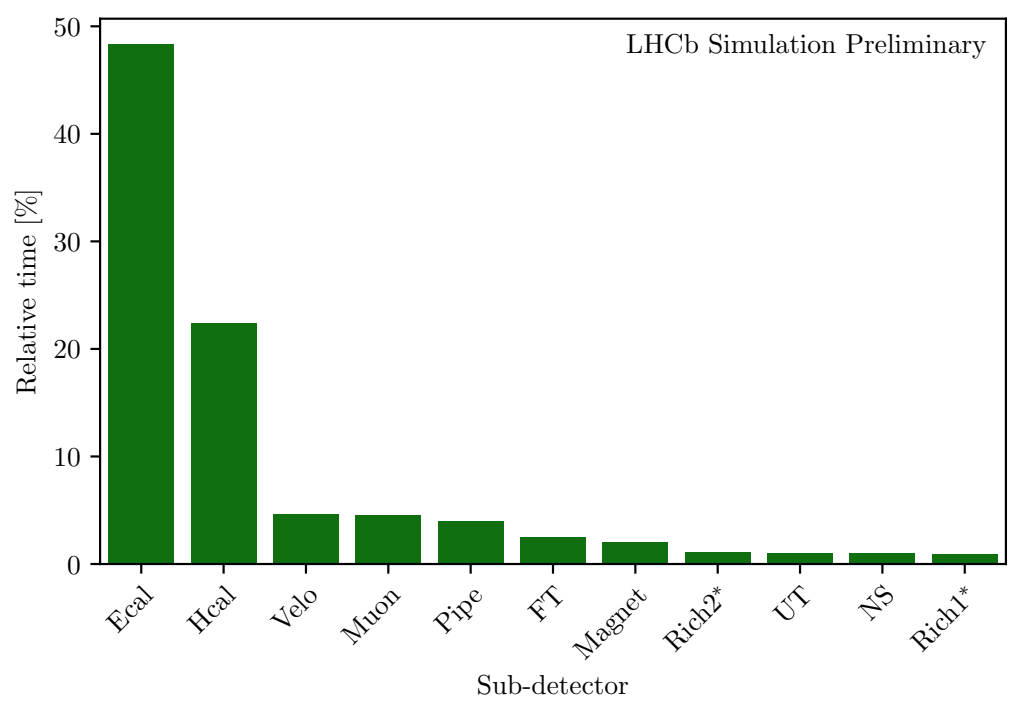

a) Current Gauss framework with Geant4 v10.6

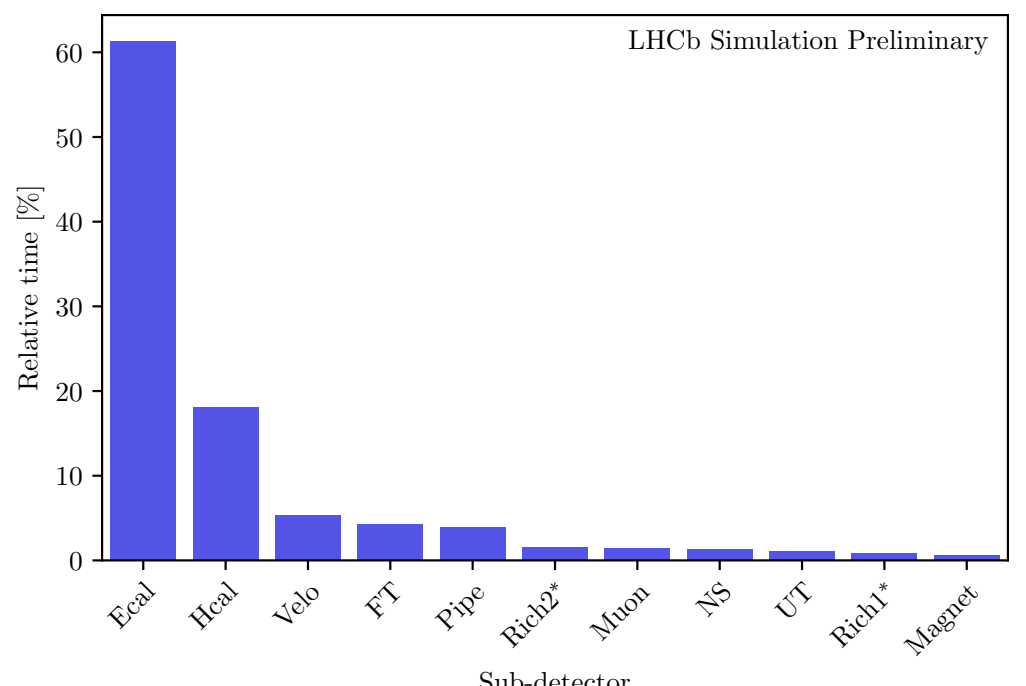

b) New multi-threaded (1 thread) Gauss-On-Gaussino framework with GEANT4 v10.7

Figure 4. Relative time [17] spent in each sub-detector when simulating 100 minimum bias events using different versions of the framework with the beam conditions as foreseen in the Run 3 data-taking period and the upgrade geometry. The time spent in the calorimeters is very similar in the current and new versions of the framework, as expected. Simulation of the optical photons in RICH1 and RICH2 was turned off in the current version of GAUss, as it is not yet available in Gauss-ON-Gaussino, for easier comparison. 


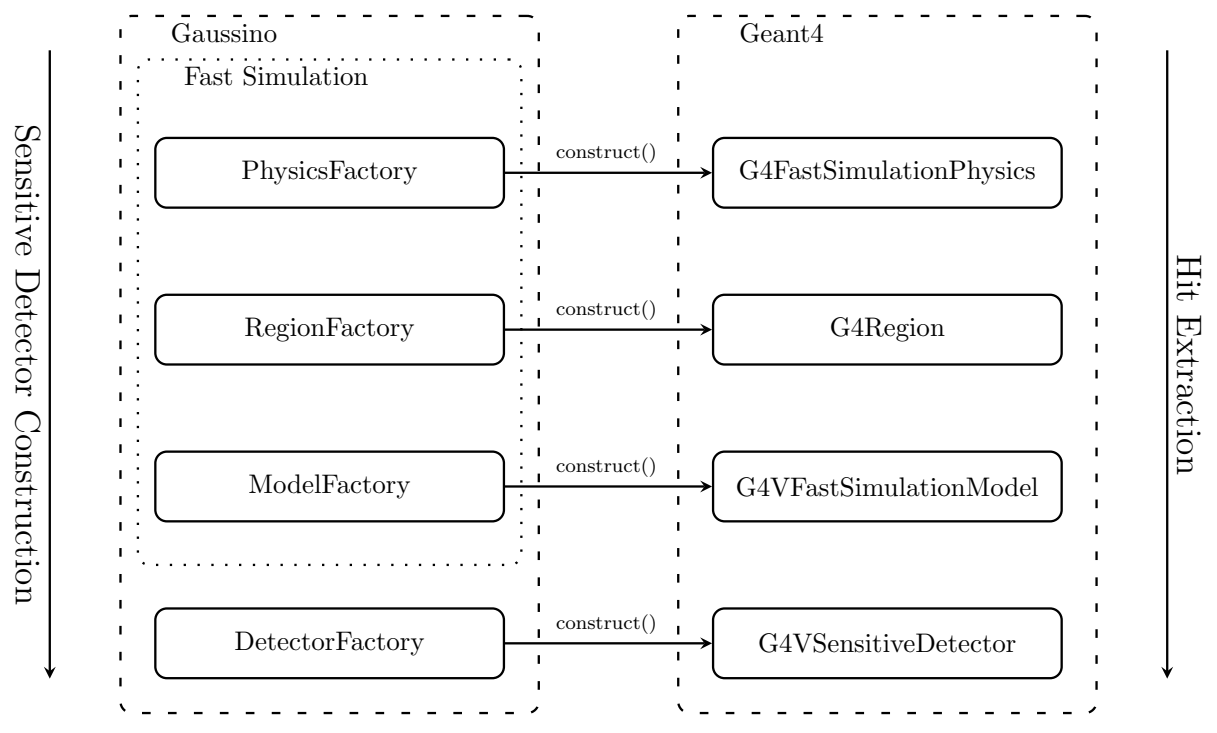

Figure 5. A simplified model [17] of the FAStSimulation interface with a set of dedicated factories that construct the corresponding GEANT4 objects

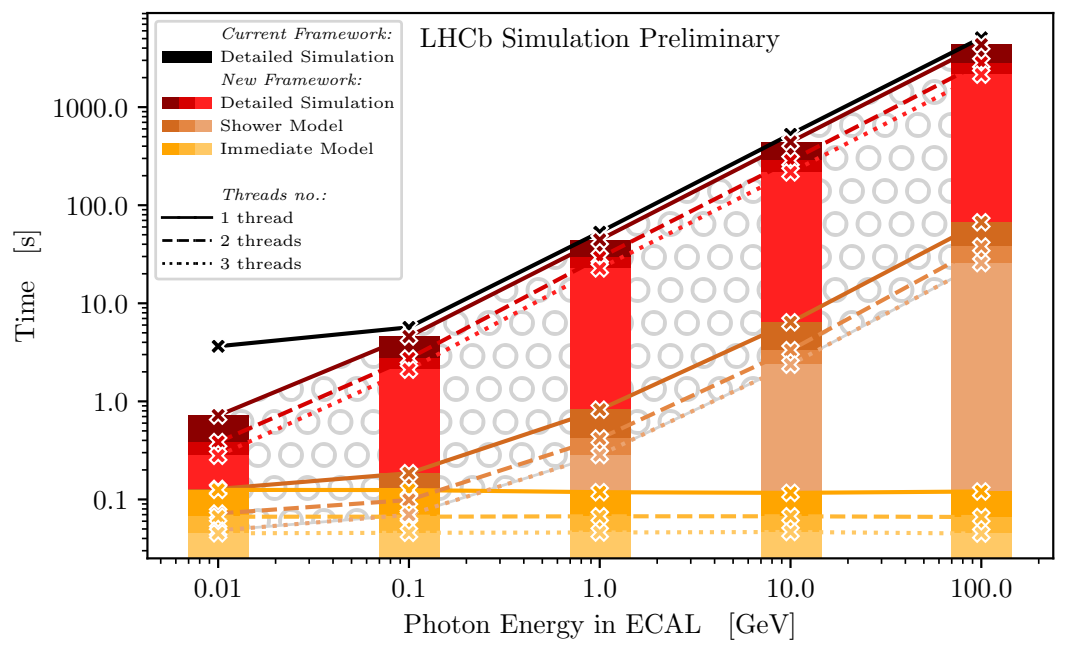

Figure 6. Comparison of the time [17] spent by different fast simulation models (ImmediateDeposit and ShowerDeposit) and a detailed simulation with GEANT4 in the electromagnetic calorimeter. In each of the models tested, a particle gun generates a grid of evenly-spaced photons of a particular energy. For the detailed simulation the time of the current version of GAuss is also given as reference. 
age in the calorimeter. Only around 25 seconds are needed for the fast simulation infrastructure in GAUSSINO with 3 threads to simulate $3328 \times 21558$ hits. In a detailed simulation, the time needed to simulate the same number of hits rises to 2145 seconds. The results prove that the infrastructure, used by the FASTSimulation interface, provides the possibility to significantly improve the time spent by the simulation software in the detector, provided the fast simulation model gives a similar level of precision in physics.

\subsection{Fast Simulation Training Datasets}

Many of the advanced, fast simulation models require prior tuning or training on some input data in order to provide valid results. When implementing a fast simulation model, a developer specifies a region that does not necessarily have to coincide with the $\mathrm{LHCb}$ sub-detectors boundaries. The information required to train these models is not always available in an output file, as GAUSs stores only the minimum amount of information required for physics studies. Therefore, the developer should be given the possibility to turn off any unneeded optimization features and to gather information at any given place in the detector.

Information about the simulated objects can be easily obtained by introducing a new sensitive sub-detector that would register hits of an abstract type with all the information needed to train the fast simulation model. A few difficulties may be encountered with this approach though. Since these detectors will only be used in just a few, specific studies, setting them up should be configurable on the fly without having to introduce them in the existing detector description, hosted in a database in an xml format. This functionality is provided by a new package in Gaussino called ExternalDetector, that allows for sub-detectors of any shape to be inserted. An example of the external plane-like detector, as seen by GEANT4, is illustrated in Figure 7. A side view of the same setup is presented in Figure 8, together with simulated particles information.

The user can also choose what kind of factories should be attached to an external detector. In principle, the external detector can become a sensitive detector (i.e., it will be able to register hits) or the user can add a monitoring tool that will be launched at the end of each event in order to verify the integrity of the collected data. MCCOLLECTOR provides a set of abstract sensitive detector factories that are easier to configure than those used in the standard simulation. Finally, it might be the case that the external detector will overlap with other existing volumes in the geometry. PARAllelGEOMETRY exploits an abstract concept introduced by GEANT4 that allows for having multiple geometries in parallel, each of them performing the particle transport without interference from objects defined in other geometries.

The packages provide a generic way of producing the training datasets for fast simulation models. Two simple examples are presented in this paper in order to show how they can be used for the models under development for ECAL (e.g. point library [21] and GANs [22]). Visual representations of the training datasets, pro- 


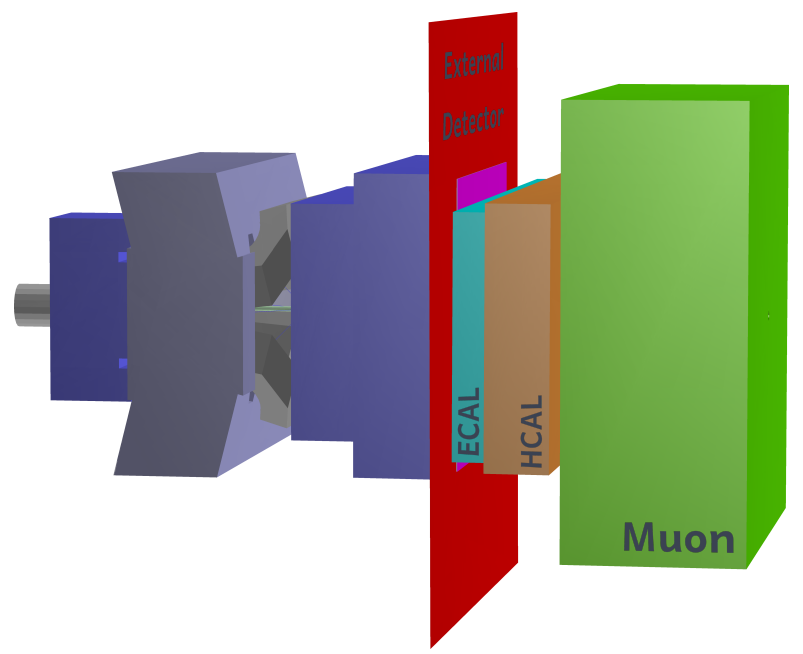

Figure 7. LHCb upgrade geometry [17] as seen by the GEANT4 toolkit with an example of a plane-like detector (red, thin plane), introduced by the ExternalDeteCtor package. When used as a collector of particle information, it may provide the source of training information about incident particles for all the sub-detectors placed downstream from it along the beamline: ECAL (cyan box), HCAL (orange box), or muon system (green box).

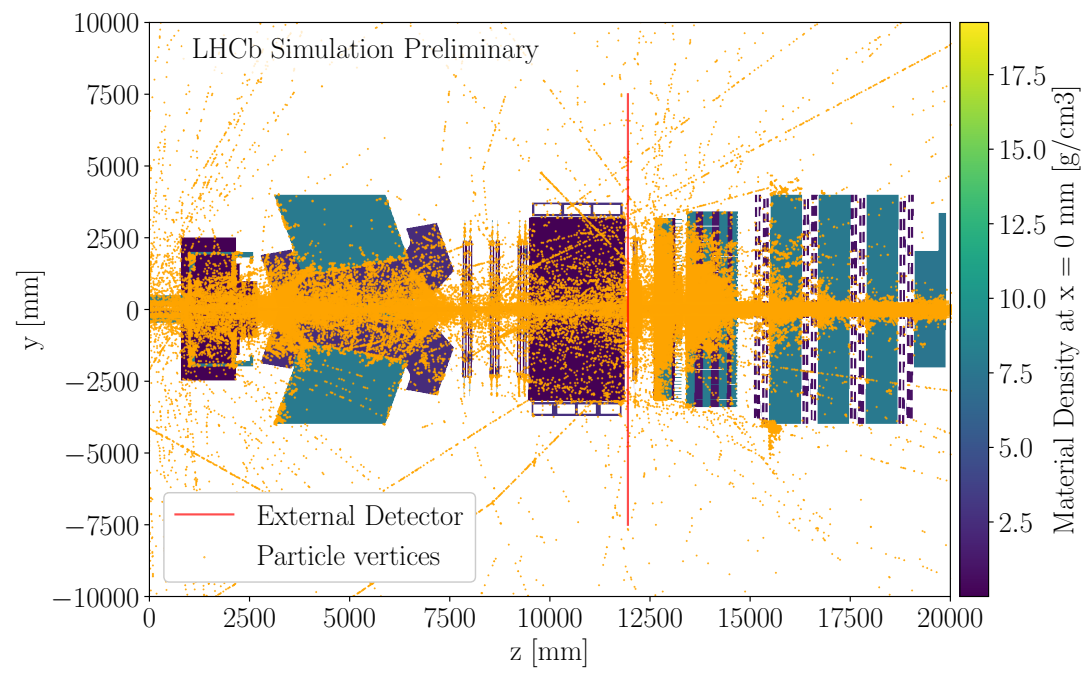

Figure 8. Particles generated [17] using the current GAuss framework in the simulation of a minimum bias event with the beam conditions, as foreseen in the Run 3 data-taking period and the upgrade geometry. An external plane-like detector that collects information about traversing particles is depicted with the red color. 


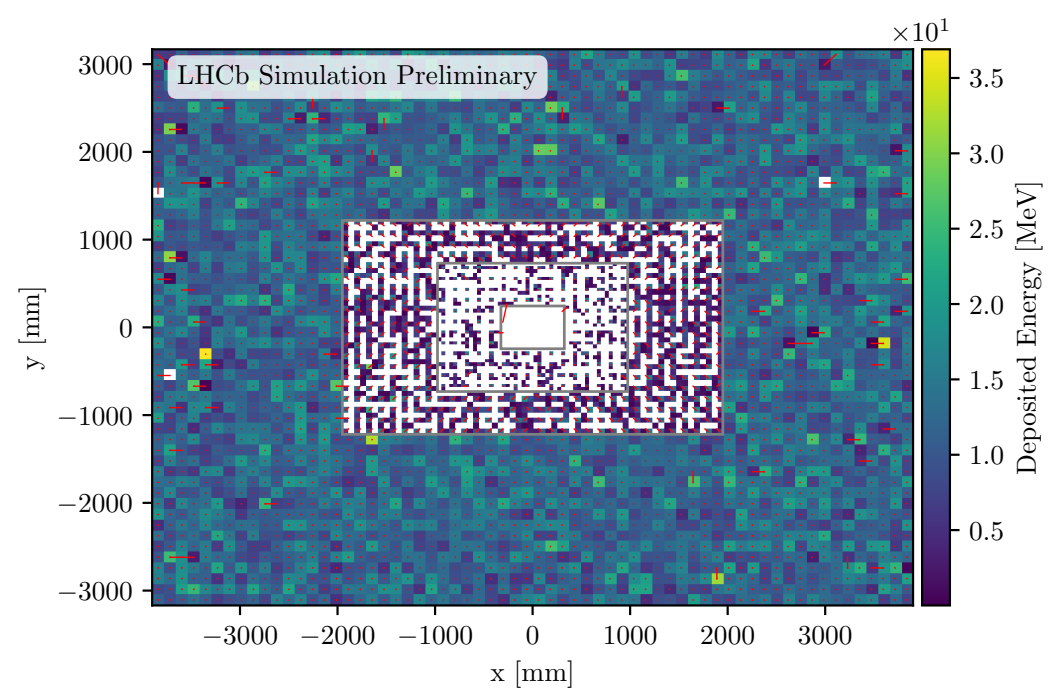

a) Particle gun with a grid of 3328 evenly-spaced $100 \mathrm{MeV}$ photons

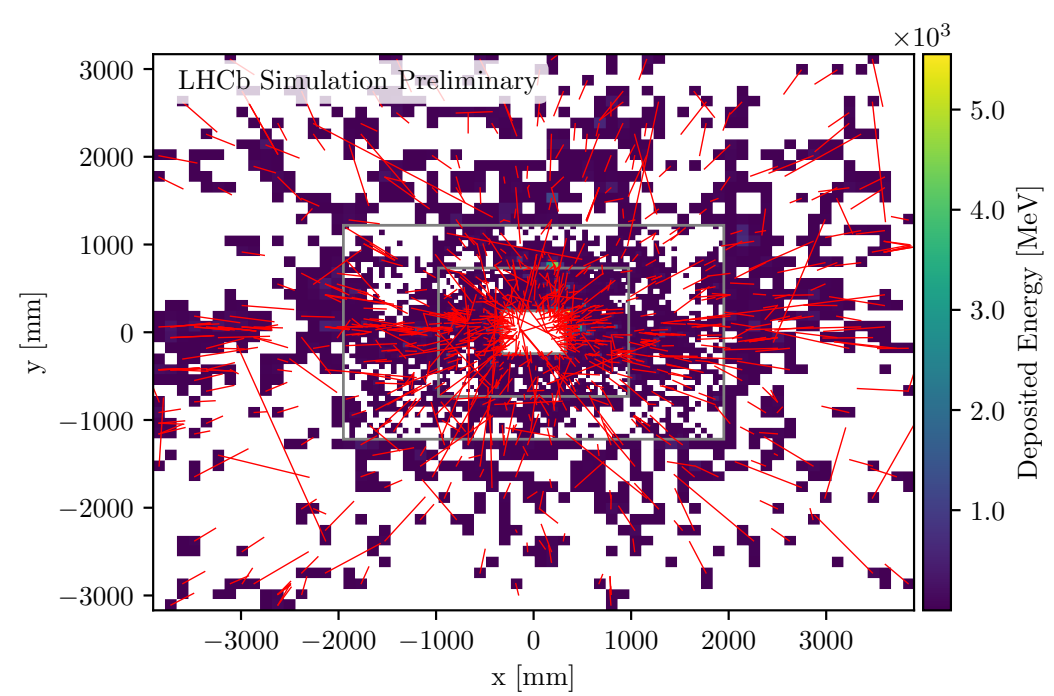

b) Minimum bias event with the beam conditions, as foreseen in the Run 3 data-taking period and the upgrade geometry

Figure 9. Visualization [17] of the training dataset produced by placing a collector plane in front of the electromagnetic calorimeter. Each of the images represent the ECAL energy deposits (hits) projected onto an $x y$-plane. The main role of the collector plane is to gather the positions of all particles intercepting the front face of ECAL. The particle positions are then linked (red lines) with the energetic centers of the showers generated by these particles. 
duced by placing a collector plane in front of ECAL, are illustrated in Figure 9, In Figure 9 a), a grid of 3328 evenly-spaced photons, similar to that used as an example when testing the performance of the interface, is shown. In Figure $9 \mathrm{~b}$ ), an input for the fast simulation studies requiring minimum bias events is presented.

This new interface will be exploited to provide training samples and integrate fast simulation models for given sub-detectors in Gaussino.

\section{CONCLUSIONS}

Monte Carlo simulation is a key component to model the effects of detector acceptance and imposed selection requirements. In order to address the computing constraints of the future upgrades of the LHCb detector, the LHCb simulation software GAUss has been redesigned to provide support for inter-event parallelism and integrate fast simulation models in GeAnT4. In this paper, a FASTSimulation interface in GAUSSINO, the new experiment independent core simulation framework developed in $\mathrm{LHCb}$, was presented alongside with performance tests that show how the multi-threaded infrastructure of the fast simulation interface has a negligible contribution to the execution time. A general way of producing the training datasets for the fast simulation models using the LHCb setup was also discussed. This new functionality will make it possible to integrate in the GAUssino framework the fast simulation models that are currently being developed in the LHCb experiment as well as future ones.

\section{REFERENCES}

[1] Abellán Beteta, C.-Alfonso Abero, A.-Amhis, Y.-Barsuk, S.Beigbeder-Beau, C. et al.: Calibration and Performance of the LHCb Calorimeters in Run 1 and 2 at the LHC. https://cds.cern.ch/record/2729028, 2020.

[2] Agostinelli, S.-Allison, J.-Amako, K.-Apostolakis, J.-Araujo, H. et al.: Geant4 - A Simulation Toolkit. Nuclear Instruments and Methods in Physics Research Section A: Accelerators, Spectrometers, Detectors and Associated Equipment, Vol. 506, 2003, No. 3, pp. 250-303, doi: 10.1016/S0168-9002(03)01368-8.

[3] Allison, J.-Amako, K.-Apostolakis, J.-Araujo, H.-Dubois, P. A. et al.: Geant4 Developments and Applications. IEEE Transactions on Nuclear Science, Vol. 53, 2006, No. 1, pp. 270-278, doi: 10.1109/TNS.2006.869826.

[4] Barrand, G.-Belyaev, I.-Binko, P.-Cattaneo, M.-Chytracek, R.Corti, G.-Frank, M.-Gracia, G.-Harvey, J.-van Herwijnen, E.Maley, P.-Mato, P.-Probst, S.-Ranjard, F.: GAUDI - A Software Architecture and Framework for Building HEP Data Processing Applications. Proceedings of CHEP 2000, Padova, Italy, 2000. Computer Physics Communications, Vol. 140, 2001, No. 1-2, pp. 45-55, doi: 10.1016/s0010-4655(01)00254-5.

[5] Belyaev, I.-Brambach, T.-Brook, N. H.-Gauvin, N.-Corti, G. et al.: Handling of the Generation of Primary Events in Gauss, the LHCb Simulation Frame- 
work. Journal of Physics: Conference Series, Vol. 331, 2011, Art. No. 032047, doi: 10.1088/1742-6596/331/3/032047

[6] Bozzi, C.-Ponce, S.-Roiser, S.: The Core Software Framework for the LHCb Upgrade. $23^{\text {rd }}$ International Conference on Computing in High Energy and Nuclear Physics (CHEP 2018), Section T5 - Software Development, 2018. EPJ Web of Conferences, Vol. 214, 2019, Art. No. 05040, doi: 10.1051/epjconf/201921405040.

[7] Clemencic, M.-Corti, G.-Easo, S.-Jones, C. R.-Miglioranzi, S. et al.: The LHCb Simulation Application, Gauss: Design, Evolution and Experience. Journal of Physics: Conference Series, Vol. 331, 2011, No. 3, Art. No. 032023, doi: 10.1088/1742-6596/331/3/032023

[8] Clemencic, M.-Degaudenzi, H.-Mato, P.-Binet, S.-Lavrijsen, W.Leggett, C.-Belyaev, I.: Recent Developments in the LHCb Software Framework Gaudi. Journal of Physics: Conference Series, Vol. 219, 2010, No. 4, Art. No. 042006, doi: 10.1088/1742-6596/219/4/042006.

[9] Frank, M.-Gaede, F.-Grefe, C.-Mato, P.: DD4hep: A Detector Description Toolkit for High Energy Physics Experiments. Journal of Physics: Conference Series, Vol. 513, 2014, No. 2, Art. No. 022010, doi: 10.1088/1742-6596/513/2/022010

[10] Lange, D. J.: The EvtGen Particle Decay Simulation Package. Nuclear Instruments and Methods in Physics Research Section A: Accelerators, Spectrometers, Detectors and Associated Equipment, Vol. 462, 2001, No. 1-2, pp. 152-155, doi: 10.1016/s01689002(01)00089-4

[11] LHCb Collaboration et al.: The LHCb Detector at the LHC. Journal of Instrumentation, Vol. 3, 2008, Art. No. S08005, doi: 10.1088/1748-0221/3/08/S08005.

[12] LHCb Collaboration: LHCb Detector Performance. International Journal of Modern Physics A, Vol. 30, 2015, No. 7, Art. No. 1530022, doi: 10.1142/S0217751X15300227.

[13] LHCb Collaboration: Computing Model of the Upgrade LHCb Experiment. CERNLHCC-2018-014, LHCB-TDR-018, Technical Design Report, LHCb, CERN, http: //cds.cern.ch/record/2319756, 2018.

[14] LHCb Collaboration. LHCb CPU Usage Forecast. Report LHCB-FIGURE-2019-018, https://cds.cern.ch/record/2696552?ln=en, 2019.

[15] LHCb Collaboration: Performance of a Multithreaded Prototype for the Future LHCb Simulation Framework (Gauss-on-Gaussino). Report LHCB-FIGURE-2019012, https://cds.cern.ch/record/2694003, 2019.

[16] LHCb Collaboration: Performance of the Lamarr Prototype: The Ultra-Fast Simulation Option Integrated in the LHCb Simulation Framework. Report LHCB-FIGURE2019-017, https://cds.cern.ch/record/2696310, 2019.

[17] LHCb Collaboration: Performance of the Fast Simulation Interface in Gausson-Gaussino. Report LHCB-FIGURE-2021-004, https://cds.cern.ch/record/ 2781378, 2021.

[18] Matev, R.: A $30 \mathrm{MHz}$ Software Trigger for the LHCb Upgrade. 23 ${ }^{\text {rd }}$ International Conference on Computing in High Energy and Nuclear Physics (CHEP 2018), Sofia, Bulgaria, 2018. Report LHCb-TALK-2018-343, https://cds.cern.ch/ record/2631781, 2018. 
[19] Müller, D.: Adopting New Technologies in the LHCb Gauss Simulation Framework. $23^{\text {rd }}$ International Conference on Computing in High Energy and Nuclear Physics (CHEP 2018), Section T2 - Offline Computing, 2018. EPJ Web of Conferences, Vol. 214, 2019, Art. No. 02004, doi: 10.1051/epjconf/201921402004.

[20] Müller, D.-Clemencic, M.-Corti, G.-Gersabeck, M.: ReDecay: A Novel Approach to Speed Up the Simulation at LHCb. The European Physical Journal C, Vol. 78, 2018, Art. No. 1009, doi: 10.1140/epjc/s10052-018-6469-6.

[21] Rama, M.-Vitali, G.: Calorimeter Fast Simulation Based on Hit Libraries LHCb Gauss Framework. $23^{\text {rd }}$ International Conference on Computing in High Energy and Nuclear Physics (CHEP 2018), Section T2 - Offline Computing, 2018. EPJ Web of Conferences, Vol. 214, 2019, Art. No. 02040, doi: 10.1051/epjconf/201921402040.

[22] Ratnikov, F.: Generative Adversarial Networks for LHCb Fast Simulation. 24 $4^{\text {th }}$ International Conference on Computing in High Energy and Nuclear Physics (CHEP 2019), Section 2 - Offline Computing, 2019. EPJ of Conferences, Vol. 245, 2020, Art. No. 02026, doi: 10.1051/epjconf/202024502026

[23] Siddi, B. G.-Müller, D.: Gaussino - A Gaudi-Based Core Simulation Framework. 2019 IEEE Nuclear Science Symposium and Medical Imaging Conference (NSS/MIC), 2019, pp. 1-4, doi: $10.1109 / \mathrm{nss} / \mathrm{mic} 42101.2019 .9060074$.

[24] Sjöstrand, T.-Mrenna, S.-Skands, P.: PYTHIA 6.4 Physics and Manual. Journal of High Energy Physics, Vol. 5, 2006, Art. No. 026, doi: 10.1088/11266708/2006/05/026.

[25] Sjöstrand, T.-Mrenna, S.-Skands, P.: A Brief Introduction to PYTHIA 8.1. Computer Physics Communications, Vol. 178, 2008, No. 11, pp. 852-867, doi: 10.1016/j.cpc.2008.01.036

[26] Whitehead, M. P.: A Palette of Fast Simulations in LHCb. XXXIX International Conference on High Energy Physics, Seoul, Korea, 2018. Report LHCb-TALK-2018302, https://cds.cern.ch/record/2630475, 2018. 

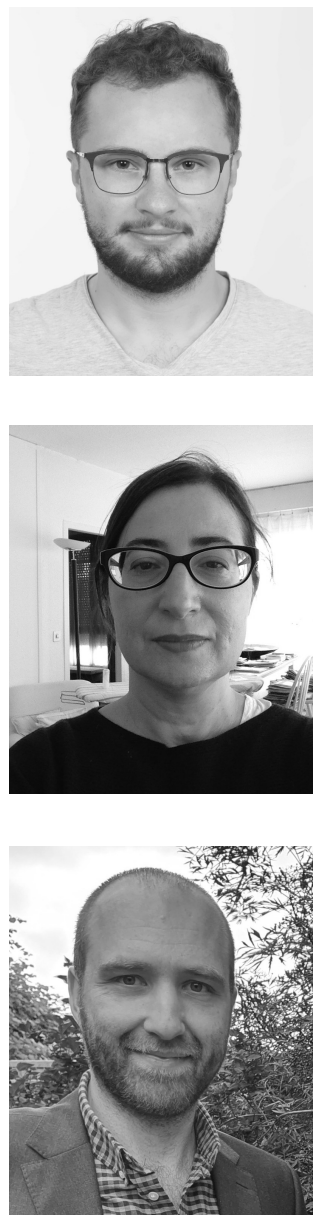

Michał Mazurex is Ph.D. student at the National Centre for Nuclear Research in Warsaw, currently at CERN in Geneva doing his Doctoral Student internship. His research interests are mathematical modelling, software engineering, data science, particle physics and machine learning.

Gloria CoRTI is senior physicist at CERN and a member of the LHCb Experiment since the beginning. She has been responsible for the LHCb simulation software for many years and continues to drive the development of various of its aspects. She has served in various software review panels and in 2020-2021 she was a convener of the HEP Software Foundation detector simulation working group.

Dominik MüLLER was Fellow at CERN in the LHCb experiment and the first main author of GAussino. He now works as a Machine Learning Engineer at FREE NOW in Hamburg. 


\section{APPENDIX}

\begin{tabular}{|c|c|c|c|c|c|c|c|c|c|c|c|}
\hline & & & & & lative & time [ & & & & & \\
\hline Velo - & 1.3 & 0.5 & 1.5 & 0.2 & $<0.1$ & 0.5 & 0.4 & $<0.1$ & $<0.1$ & 0.2 & \\
\hline Rich1* & 0.2 & 0.4 & 0.1 & $<0.1$ & $<0.1$ & $<0.1$ & 0.2 & $<0.1$ & $<0.1$ & $<0.1$ & \\
\hline $\mathrm{UT}-$ & 0.2 & 0.4 & 0.2 & $<0.1$ & $<0.1$ & $<0.1$ & $<0.1$ & $<0.1$ & $<0.1$ & $<0.1$ & \\
\hline Magnet - & 0.4 & 0.7 & 0.1 & $<0.1$ & $<0.1$ & $<0.1$ & 0.6 & $<0.1$ & $<0.1$ & $<0.1$ & \\
\hline FT & 0.9 & 0.6 & 0.4 & $<0.1$ & $<0.1$ & $<0.1$ & 0.4 & $<0.1$ & $<0.1$ & $<0.1$ & \\
\hline $\operatorname{Rich} 2^{*}$ & 0.3 & 0.4 & 0.1 & $<0.1$ & $<0.1$ & $<0.1$ & 0.2 & $<0.1$ & $<0.1$ & $<0.1$ & \\
\hline NS & 0.2 & 0.3 & $<0.1$ & $<0.1$ & $<0.1$ & $<0.1$ & 0.3 & $<0.1$ & $<0.1$ & $<0.1$ & \\
\hline Ecal & 18.7 & 17.5 & 1.9 & 0.2 & $<0.1$ & 0.7 & 8.6 & $<0.1$ & $<0.1$ & 0.5 & \\
\hline Hcal - & 4.5 & 4.4 & 1.0 & $<0.1$ & $<0.1$ & 0.6 & 11.1 & $<0.1$ & $<0.1$ & 0.6 & \\
\hline Muon & 0.7 & 1.0 & 0.1 & $<0.1$ & $<0.1$ & 0.1 & 2.3 & $<0.1$ & $<0.1$ & 0.1 & \\
\hline Pipe - & 1.2 & 1.6 & 0.2 & $<0.1$ & $<0.1$ & $<0.1$ & 0.9 & $<0.1$ & $<0.1$ & $<0.1$ & \\
\hline
\end{tabular}

a) Relative time spent by each particle in a sub-detector measured with respect to the total time spent on the simulation

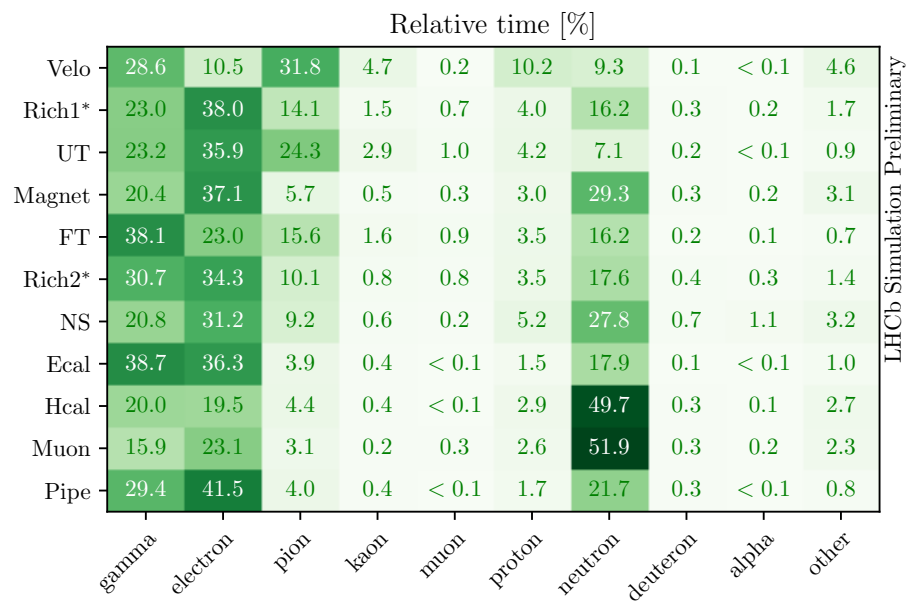

b) Relative time by each particle in a sub-detector measured with respect to the time spent in that sub-detector

Figure 10. Performance [17] of the current Gauss framework when simulating 100 minimum bias events with the beam conditions, as foreseen in the Run 3 data-taking period and the upgrade geometry 


\begin{tabular}{|c|c|c|c|c|c|c|c|c|c|c|c|}
\hline & & & & & Urv & ime [ & & & & & \\
\hline Velo - & 2.0 & 0.8 & 1.6 & 0.3 & $<0.1$ & 0.2 & 0.2 & $<0.1$ & $<0.1$ & 0.4 & \\
\hline Rich1* & 0.3 & 0.3 & 0.1 & $<0.1$ & $<0.1$ & $<0.1$ & $<0.1$ & $<0.1$ & $<0.1$ & $<0.1$ & \\
\hline $\mathrm{UT}$ & 0.4 & 0.3 & 0.2 & $<0.1$ & $<0.1$ & $<0.1$ & $<0.1$ & $<0.1$ & $<0.1$ & $<0.1$ & \\
\hline Magnet & 0.3 & 0.2 & $<0.1$ & $<0.1$ & $<0.1$ & $<0.1$ & $<0.1$ & $<0.1$ & $<0.1$ & $<0.1$ & \\
\hline FT & 2.1 & 1.4 & 0.4 & $<0.1$ & $<0.1$ & $<0.1$ & 0.2 & $<0.1$ & $<0.1$ & $<0.1$ & \\
\hline $\operatorname{Rich} 2^{*}$ & 0.6 & 0.5 & 0.1 & $<0.1$ & $<0.1$ & $<0.1$ & 0.1 & $<0.1$ & $<0.1$ & $<0.1$ & \\
\hline NS & 0.4 & 0.6 & 0.1 & $<0.1$ & $<0.1$ & $<0.1$ & 0.1 & $<0.1$ & $<0.1$ & $<0.1$ & \\
\hline Ecal & 30.2 & 23.5 & 2.1 & 0.3 & $<0.1$ & 0.9 & 3.6 & $<0.1$ & $<0.1$ & 0.5 & \\
\hline Hcal - & 5.5 & 4.6 & 1.2 & $<0.1$ & $<0.1$ & 0.8 & 5.1 & $<0.1$ & $<0.1$ & 0.7 & \\
\hline Muon & 0.4 & 0.4 & $<0.1$ & $<0.1$ & $<0.1$ & $<0.1$ & 0.4 & $<0.1$ & $<0.1$ & $<0.1$ & \\
\hline Pipe & 1.8 & 1.7 & 0.1 & $<0.1$ & $<0.1$ & $<0.1$ & 0.2 & $<0.1$ & $<0.1$ & $<0.1$ & \\
\hline
\end{tabular}

a) Relative time by each particle in a sub-detector measured with respect to the total time spent on the simulation

\begin{tabular}{|c|c|c|c|c|c|c|c|c|c|c|c|}
\hline & \multicolumn{11}{|c|}{ Relative time $[\%]$} \\
\hline Velo & 36.7 & 14.4 & 29.8 & 5.0 & 0.1 & 2.9 & 3.3 & 0.1 & $<0.1$ & 7.8 & \\
\hline Rich1* & 32.7 & 31.5 & 15.2 & 2.7 & 0.6 & 3.6 & 9.7 & 0.5 & 0.2 & 3.4 & \\
\hline UT & 40.8 & 27.9 & 18.2 & 3.2 & 0.7 & 2.5 & 2.6 & $<0.1$ & $<0.1$ & 3.9 & \\
\hline Magnet & 43.4 & 39.5 & 6.5 & 0.2 & $<0.1$ & 1.6 & 1.7 & 0.3 & 0.2 & 6.6 & \\
\hline $\mathrm{FT}$ & 48.6 & 32.5 & 9.0 & 1.2 & 0.9 & 1.6 & 4.0 & 0.2 & $<0.1$ & 1.9 & \\
\hline Rich2* & 42.1 & 35.7 & 7.8 & 0.9 & 0.7 & 2.5 & 7.4 & 0.5 & 0.2 & 2.1 & \\
\hline NS & 32.1 & 40.8 & 7.6 & 0.8 & 0.2 & 4.1 & 10.1 & 0.7 & 0.7 & 2.8 & \\
\hline Ecal & 49.2 & 38.4 & 3.4 & 0.4 & 0.1 & 1.4 & 5.9 & 0.2 & $<0.1$ & 0.9 & \\
\hline Hcal & 30.4 & 25.4 & 6.4 & 0.2 & 0.2 & 4.6 & 28.1 & 0.5 & 0.2 & 4.0 & \\
\hline Muon & 25.6 & 27.9 & 5.5 & 0.6 & 0.8 & 5.5 & 30.3 & 0.5 & 0.1 & 3.2 & \\
\hline Pipe & 45.8 & 43.4 & 2.9 & $<0.1$ & $<0.1$ & 1.7 & 4.0 & 0.4 & $<0.1$ & 1.6 & \\
\hline
\end{tabular}

b) Relative time by each particle in a sub-detector measured with respect to the time spent in that sub-detector

Figure 11. Performance [17] of the new multi-threaded (1 thread) Gaussino-based framework when simulating 100 minimum bias events with the beam conditions, as foreseen in the Run 3 data-taking period and the upgrade geometry 